\title{
BINAURAL CHARACTERISTICS OF UNITS IN THE OWL'S BRAINSTEM AUDITORY PATHWAY: PRECURSORS OF RESTRICTED SPATIAL RECEPTIVE FIELDS ${ }^{1}$
}

\author{
A. MOISEFF ${ }^{2}$ AND M. KONISHI
}

Division of Biology, 216-76, California Institute of Technology, Pasadena, California 91125

Received December 13, 1982; Revised June 27, 1983; Accepted June 28, 1983

\begin{abstract}
The barn owl uses binaural phase and intensity differences for sound localization. These two cues also determine the receptive fields of specialized neurons in the inferior colliculus. The main aim of this study was to investigate where neuronal sensitivity to the binaural cues emerges in the brainstem auditory nuclei, and how this sensitivity reaches the neurons in the inferior colliculus. The owl's phase-sensitive neurons are selective to microsecond phase differences of high frequency signals, unlike mammalian phase-sensitive neurons which are restricted to low frequency signals. In certain nuclei virtually all of the neurons are sensitive to either phase differences or intensity differences, but not to both. These nuclei form two distinctly separate pathways that converge at the inferior colliculus where neurons selective to both phase and intensity differences occur. In contrast to the mammalian auditory system, the owl's phase- and intensity difference-sensitive pathways are not segregated into low frequency and high frequency channels.
\end{abstract}

The barn owl's auditory midbrain contains neurons that have spatial receptive fields: they respond only to sounds located in a single, restricted region of space (Knudsen and Konishi, 1978a, b). This property must be the result of neural processing of binaural information (Knudsen and Konishi, 1982). An "image" of auditory space does not project directly onto the sensory epithelium of the cochlea (which would be analogous to visual space being projected onto the retina); rather, the cochlea maps frequency along its length. Location can, however, be extracted from binaural cues. The interaural difference in the arrival time of sound is determined predominantly by the position of the source along the azimuthal axis. The elevation of the sound source affects the intensity of the sound reaching the vertically asymmetric ears of the owl, resulting in a dependence of interaural intensity difference on elevation. With appropriate neural processing, sound location can be determined from these binaural cues.

The receptive fields of space-specific units (auditory

\footnotetext{
${ }^{1}$ We thank Eugene Akutagawa for histological assistance, Herb Adams for constructing a new stereotaxic device, and Mike Walsh and his staff for their help in designing and maintaining much of our instrumentation. We appreciate the helpful comments of Eric Knudsen and Terry Takahashi. This work was supported by National Institutes of Health Grant NS14617 to M. K. and Helen Hay Whitney and Del E. Webb postdoctoral fellowships to A. M.

${ }^{2}$ To whom correspondence should be addressed, at Biological Sciences Group, U-42, University of Connecticut, Storrs, CT 06268.
}

units with restricted spatial receptive fields) are attributed to the neurons being excited only by a narrow range of both interaural phase (time) delay and interaural intensity differences (Moiseff and Konishi, 1981a). If either of these differences is beyond a certain range, the unit will fail to respond or, in some cases, will be inhibited. Therefore, in our survey of the functional properties of the brainstem nuclei, we were primarily interested in determining how and where sensitivity to interaural phase and intensity differences emerges in the ascending pathway.

\section{Materials and Methods}

The techniques employed in this study were similar to those of an earlier study (Moiseff and Konishi, 1981a) and will be summarized only briefly. Adult barn owls ( Tyto alba) were prepared for neurophysiological recordings by removing small areas of skull while anesthetized (Ketaset, Bristol Laboratories, $4 \mathrm{mg} / \mathrm{kg}$ ). Anesthesia was maintained throughout the recording sessions by periodic injections. A heating pad was used to maintain the animal's normal body temperature.

Platinum-iridium or Elgiloy microelectrodes, electrolytically sharpened and insulated with glass (Wolbarsht et al., 1960), or glass micropipettes (filled with $0.5 \mathrm{M}$ sodium acetate, or $2 \%$ horseradish peroxidase (HRP) in $0.5 \mathrm{M} \mathrm{KCl}$ ) were positioned stereotaxically. Electrode tracks were marked by electrolytic lesions or iontophor- 
etic ejection of HRP. Lesions were identified in cresyl violet-stained sections, and HRP ejection sites were visualized in unstained or neutral red counterstained sections (Mesulam, 1978). The clarity of lesions in Figures 3 and 6 is due to darkly stained sites of gliosis shown in high contrast prints. The anatomical connections of the brainstem nuclei will be reported in a separate article ( $T$. Takahashi and M. Konishi, manuscript in preparation).

Auditory stimuli consisting of tone bursts or bandpassed pseudorandonı noise (center frequency $7 \mathrm{kHz}, 15$ $\mathrm{dB}$ /octave rolloff) were delivered dichotically through magnetic earphones or condenser transducers (Knowles BT 1759) that were installed in the owl's ear canals. Bursts were 100 to $120 \mathrm{msec}$ long and had rise/fall times of $0.5 \mathrm{msec}$. Digital attenuators and a digital phase shifter controlled the interaural intensity difference (IID) and the interaural phase disparity (ongoing time disparity (OTD)). Sound pressure levels of tonal stimuli were measured with a probe microphone (Bruel \& Kjaer 12.5$\mathrm{cm}$ microphone with $1.2-\mathrm{mm}$ diameter probe tube) surgically implanted into the ear canal, the probe microphone having been previously calibrated under free-field conditions. The RMS voltage of noise stimuli was adjusted to that of calibrated tonal stimuli. All experiments were carried out in a $5 \times 3 \times 3 \mathrm{~m}$ anechoic, soundattenuated chamber.

Extracellular action potentials ("spikes") were converted to computer (PDP-11/40)-compatible signals with a window discriminator (Ortec model 730). A real time clock (Digital KW11-K) measured the time of occurrence of the discriminator output and stored this value on disk for subsequent analysis. Response latencies reported in this paper are the minimum latencies obtained in the particular nuclei. Latencies of binaural units were measured in response to binaural stimulation at the optimal IID and OTD (rise/fall time of the stimulus was set to $0.5 \mathrm{msec}$ ).

All units were subjected to a standard test protocol that included both monaural and binaural stimulation. The mean number of spikes per stimulus presentation (spike count) served as a measure of a unit's response to all tests. Responses to monaural stimulation were classified according to the scheme described under "Results." Best frequencies were determined with monaural stimuli. Ail units with best frequencies above $4 \mathrm{kHz}$, even those that appeared to receive only monaural input, were then presented with binaural stimuli for the test of sensitivity to binaural intensity and/or phase differences. Responses to binaural phase differences were tested at a sound level 10 to $20 \mathrm{~dB}$ above the units' threshold for each ear. In this test, spike counts were obtained for at least 11 values of phase (ongoing time) disparity. Finally, units were tested for sensitivity to variations in IIDs at their optimal OTDs. In this paper the term "binaural phase difference" refers to the binaural phase difference of tonal stimuli as well as the ongoing time disparity of noise stimuli. Phase differences are expressed as characteristic delays in microseconds.

\section{Results}

The results reported here are based on 902 units isolated in the medulla and midbrain. Since our goal was an analysis of the binaural properties of auditory brainstem units, we did not record from the cochlear nuclei, nucleus angularis, and nucleus magnocellularis, which are monaural (Boord and Rasmussen, 1963; Boord, 1968). The predominant neural response properties of various nuclei will be described below. Rare response classes will not be discussed in detail, but are included in the tabulated summary of response properties (Table I). We will use the terminology of Karten and Hodos (1967) and Leibler (1975) in our description of the brainstem nuclei (Fig. 1).

\section{Monaural responses}

The use of earphones enabled us to present stimuli separately to the two ears. Because of the presence of an air-filled pathway between the owl's middle ears, monaural isolation could only be assured above certain frequencies (Moiseff and Konishi, 1981b). The interaural pathway permits appreciable acoustical crosstalk between middle ears at frequencies below about $4 \mathrm{kHz}$; the middle ears are acoustically isolated above this frequency range (attenuation by the interaural pathway exceeds 30 $\mathrm{dB}$ above $4 \mathrm{kHz}$ ). Thus, responses to monaural stimulation were obtained only from units whose best frequencies were $4 \mathrm{kHz}$ or higher.

Monaural stimuli can either excite or inhibit a unit, or have no noticeable effect on its discharge. In extracellular recordings, inhibitory effects can only be seen when a unit is spontaneously active or if a unit shows clear evidence of postinhibitory rebound. When neither of these conditions were met, inhibition and "no effect"

\section{TABLE I}

Summary of brainstem auditory nuclei units' response characteristics

The number of units within a given nucleus exhibiting the given characteristics is expressed as a percentage of total units recorded from the nucleus. Failure of some columns to add up to exactly $100 \%$ reflects round off errors in the computation of percentages. All of the 98 units recorded from the external nucleus of MLD were either "II," "IO," "OI," or "OO," and were both OTD sensitive and IID tuned. Abbreviations are the same as described in the legend to Figure 1.

\begin{tabular}{|c|c|c|c|c|c|}
\hline \multirow{3}{*}{ Classification } & \multicolumn{5}{|c|}{ Nucleus and Number of Units } \\
\hline & os & LLv & VLV & $\mathrm{La}$ & MLD \\
\hline & 291 & 118 & 297 & 98 & 98 \\
\hline \multicolumn{6}{|l|}{ EE } \\
\hline $\begin{array}{l}\text { OTD-sensitive } \\
\text { IID-insensitive }\end{array}$ & $3 \%$ & $0 \%$ & $42 \%$ & $38 \%$ & $0 \%$ \\
\hline \multicolumn{6}{|l|}{ EE } \\
\hline $\begin{array}{l}\text { OTD-insensitive } \\
\text { IID-insensitive }\end{array}$ & $23 \%$ & $0 \%$ & $4 \%$ & $40 \%$ & $0 \%$ \\
\hline \multicolumn{6}{|l|}{ EO } \\
\hline $\begin{array}{l}\text { OTD-insensitive } \\
\text { IID-insensitive }\end{array}$ & $3 \%$ & $100 \%$ & $2 \%$ & $3 \%$ & $0 \%$ \\
\hline \multicolumn{6}{|l|}{$\mathrm{OE}$} \\
\hline $\begin{array}{l}\text { OTD-insensitive } \\
\text { IID-insensitive }\end{array}$ & $72 \%$ & $0 \%$ & $0 \%$ & $9 \%$ & $0 \%$ \\
\hline \multicolumn{6}{|l|}{ EI } \\
\hline $\begin{array}{l}\text { OTD-insensitive } \\
\text { IID-sensitive }\end{array}$ & $0 \%$ & $0 \%$ & $49 \%$ & $8 \%$ & $0 \%$ \\
\hline \multicolumn{6}{|l|}{ EI } \\
\hline $\begin{array}{l}\text { OTD-sensitive } \\
\text { IID-sensitive }\end{array}$ & $0 \%$ & $0 \%$ & $0 \%$ & $0 \%$ & $0 \%$ \\
\hline
\end{tabular}




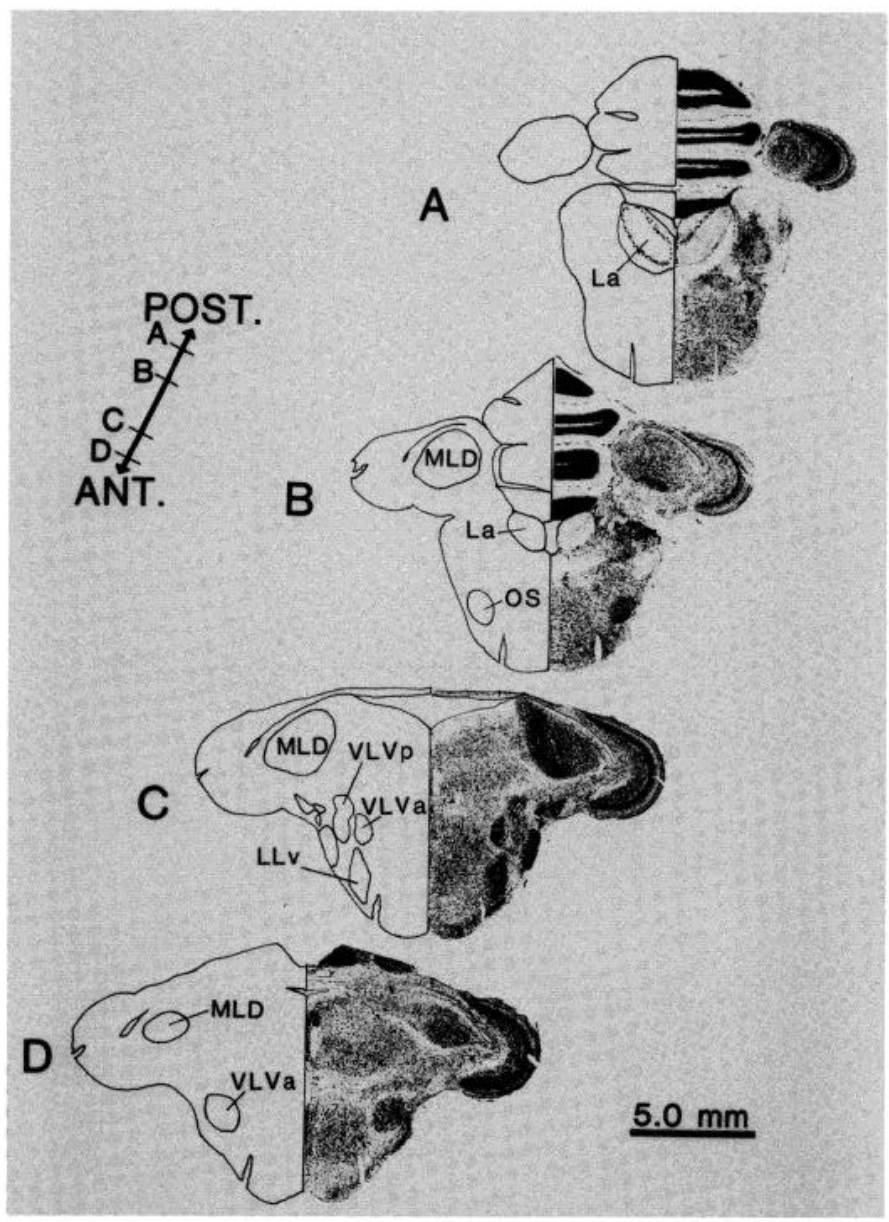

Figure 1. Auditory nuclei in the owl's brainstem. Auditory nuclei above the level of the cochlear nuclei (nucleus angularis and nucleus magnocellularis) are shown in coronal sections. Sections were stained with cresyl violet and printed at high contrast. The scale bar is for both distance within a section and distance between adjoining sections. With the exception of LLv, all nuclei contain binaural neurons. $L a$, nucleus laminaris; $L L v$, nucleus lemnisci lateralis, pars ventralis; $M L D$, nucleus mesencephalicus lateralis, pars dorsalis; $O S$, nucleus olivaris superior; $V L V$, nucleus ventralis lemnisci lateralis ( $a$, anterior, $p$, posterior).

could not be discriminated and we regarded the stimulus as having "no effect."

All neurons in the brainstem nuclei responsed to monaural stimulation. Their responses will be described by the conventional shorthand method with a pair of letters indicating the neuron's response to contralateral and ipsilateral stimulation in that order; thus, for example, "EI" stands for an excitatory response to contralateral stimulation and an inhibitory response to ipsilateral stimulation. Similarly, "OE" indicates "no effect" by contralateral stimulation and an excitatory response to ipsilateral stimulation.

\section{Binaural interactions}

When binaural units were stimulated monaurally, the most common response types were EE and EI in the brainstem and additional types were II and $\mathrm{OO}$ in the inferior colliculus. Full characterization of binaural neurons requires tests with additional stimulus variables which are unique to dichotic stimulation. These are binaural phase and intensity differences.

Units were considered to be sensitive to interaural phase differences if their spike counts for any phase tested decreased to $75 \%$ of the maximum response. Units were considered to be insensitive to phase differences if their spike counts did not fall below the $75 \%$ level at any phase difference tested.

The responses of units to binaural intensity differences showed three different patterns: insensitive, sensitive, and tuned. These were discerned by the mode of change in a unit's spike count in response to variations in either the interaural intensity difference (IID = contralateral intensity - ipsilateral intensity) or the average binaural intensity $(\mathrm{ABI}=$ contralateral intensity + ipsilateral intensity, divided by 2). When one of these parameters was varied, the other was kept constant.

To facilitate a direct comparison of neuronal response properties from different nuclei, spike counts were plotted against intensity or phase parameters in the same format throughout this report. Each point and vertical bar in the spike count curves indicates, respectively, the mean number of spikes and the standard deviation. Each figure with spike count curves illustrates the response properties of a selected neuron whose monaural and binaural responses were characteristic of the nucleus in which it was found.

\section{Response characteristics of brainstem auditory nuclei}

Nucleus olivaris superior (OS). Of 291 units recorded from $\mathrm{OS}, 72 \%$ were monaural ("OE," responded only to ipsilateral stimulation) and $23 \%$ were binaural ("EE," were excited by stimulation of either ear). These relative percentages must be interpreted with care. We used certain physiologically identifiable aspects of the $\mathrm{OE}$ area to calibrate our stereotaxic coordinates. When we found this area, we confirmed it by recording as many cells as possible from the penetration. When the penetration was completed, we made corrections in our stereotaxic coordinates to enable us to reposition the electrode in other nuclei. Thus, because our sampling of OS cells was biased for $\mathrm{OE}$ units, the relative percentages merely indicate that two classes of units occur in this nucleus, but not that there are nearly 3 times as many monaural units as binaural units. The monaural $(\mathrm{OE})$ units were located in the lateral and posterior regions of $\mathrm{OS}$, and were arranged in a clear tonotopic sequence. The best frequencies of $\mathrm{OE}$ units increased as the electrode passed deeper (more ventral) into the nucleus. Although we did not undertake a rigorous survey of the frequency representation in this area, we observed best frequencies as low as $700 \mathrm{~Hz}$ and as high as $9.1 \mathrm{kHz}$.

Electrode penetrations located more centrally and medially within the nucleus revealed units receiving binaural input. These units were excited by stimulation of either ear (Fig. 2A). Monaural response functions for the two ears were usually very similar, although we observed some response functions that differed. When the ABI was varied and the IID was kept constant, spike count increased monotonically with increasing average intensity (Fig. $2 B$ ). The binaural response was approximately equal to the sum of the monaural responses for any given 

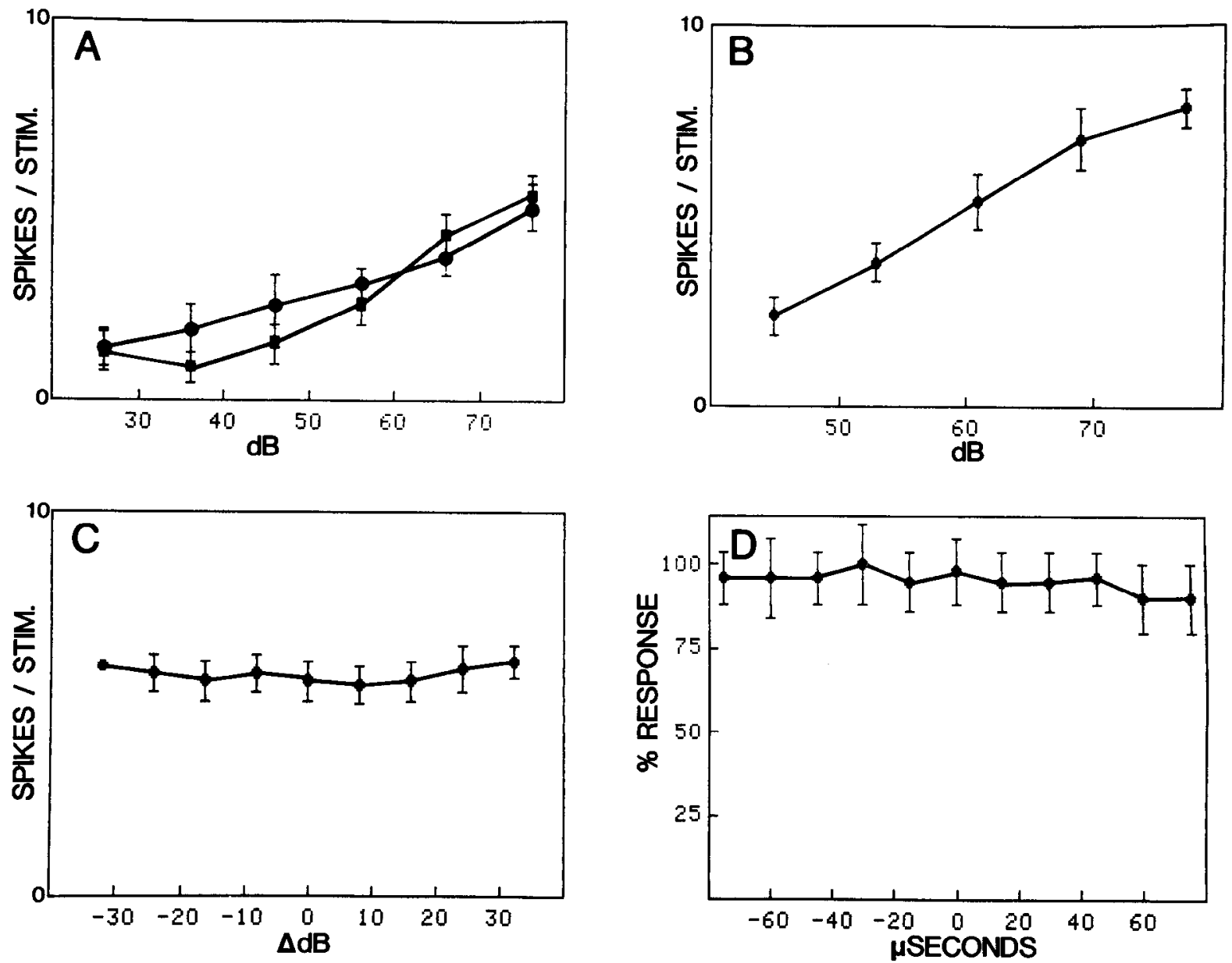

Figure 2. Response characteristics of a typical binaural OS unit. A, Stimulation of the contralateral (solid circles) ear or the ipsilateral (solid squares) ear excited the unit. $B$, The response increased with increasing ABI. The intensity at the two ears was kept equal (IID $=0$ ) and the binaural phase difference was kept at $0 \mu \mathrm{sec} . C$, The response was not modulated by varying the IID (with average intensity $=61 \mathrm{~dB}$, phase difference $=0)$. Negative values of $(\Delta \mathrm{dB}$ indicate that ipsilateral stimulation was louder than contralateral stimulation. $D$, The unit's response was insensitive to variations in the binaural phase difference from $-75 \mu \mathrm{sec}$ (contralateral ear lending) to $+75 \mu \mathrm{sec}$ (ipsilateral ear leading). The binaural intensity difference was $0 \mathrm{~dB}$; the $\mathrm{ABI}$ was $61 \mathrm{~dB}$. Stimulus: bandpassed noise. Stimulus repetitions: $8(A$ to $C)$ and $12(D)$. Spontaneous level: 1.5 spikes/stimulus $(35 \%$ in $D)$.

intensity. Varying the binaural intensity difference while keeping the average interaural intensity constant (Fig. $2 C$ ) had little effect on the magnitude of their response. The flatness of this curve reflects the "balanced" nature of the monaural inputs (Fig. 2A). These units were insensitive to binaural phase differences (Fig. $2 D$ ); the response did not fall to $75 \%$ of maximum at any value of binaural phase difference. Binaural OS units are therefore sensitive to neither binaural intensity nor phase differences. Binaural units responded at an average latency of about $3.7 \mathrm{msec}$ (range 3.1 to $5.0 \mathrm{msec}$ ).

Nucleus laminaris. Cell bodies in nucleus laminaris are large (approximately $15 \mu \mathrm{m}$ ) and widely spaced. Since the sparse distribution of cell bodies made the probability of coming close to a cell body low in any given penetration, we recorded not from within the confines of the nucleus itself, but rather from the large bundle of output fibers which exited from it (Fig. 3). Tritiated proline, injected into the nucleus, demonstrated that laminaris axons exit exclusively through this bundle (unpublished data).
We found several classes of units in the laminaris output bundle. The characteristic frequencies of the units recorded in this area ranged from about 4.5 to $8 \mathrm{kHz}$. Of the 98 units recorded, $40 \%$ were excited by monaural stimulation of either ear. The responses of these units paralleled those of the binaural OS units, just described. Responses to binaural stimuli increased when the ABI was increased, but they were insensitive to IIDs. These units were also insensitive to binaural phase differences.

Another class, comprising $38 \%$ of the units recorded, was excited by stimulation of either ear (Fig. 4 4 ) but was sensitive to binaural phase differences (Fig. $4 D$ ). Responses to binaural stimulation increased monotonically with increasing average intensity (Fig. $4 B$ ). Unlike EE units described earlier (cf. Fig. 2), the binaural response was greater than the sum of the two monaural responses. The binaural response was modulated only slightly by variations in the IID (Fig. $4 C$ ). The modulation was due to decreased spike counts as the stimulus intensity decreased toward the threshold of one of the ears. The degree of phase sensitivity among these units 


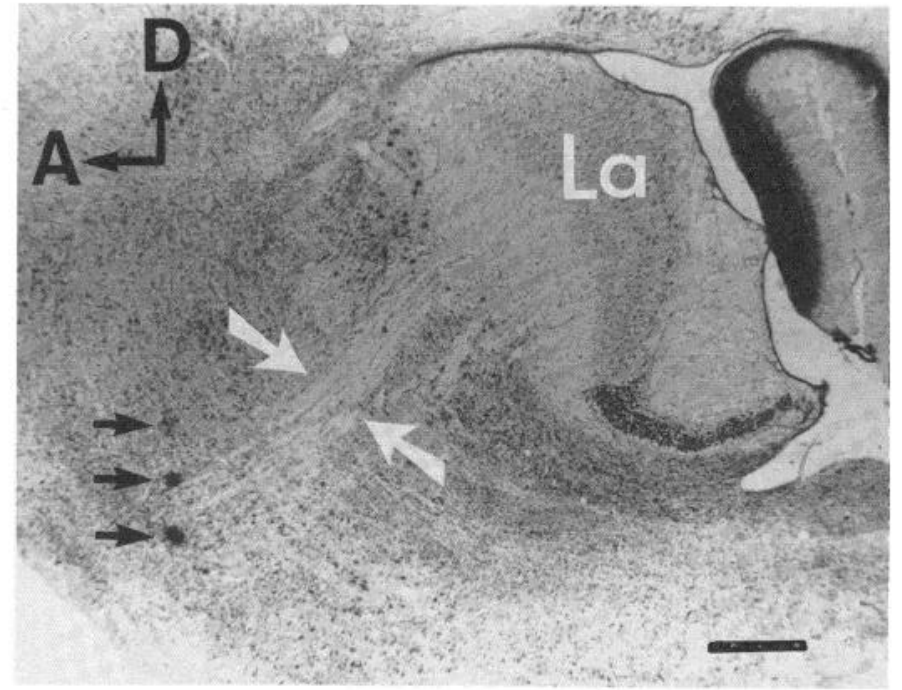

Figure 3. Nucleus laminaris output fiber bundle shown in parasagittal section. The fiber bundle is bracketed by the white arrows. Three lesions (black arrows) show a portion of an electrode track. In this particular electrode track, auditory units were recorded between the middle and lowest lesions. $L a$, cellular area of nucleus laminaris. Calibration scale $=1.0 \mathrm{~mm}$. varied widely (Fig. 5). It is not yet clear whether this variation of selectivity is distributed along a continuum, or represents separate classes of phase-sensitive laminaris units.

Within the laminaris output bundle we also encountered other classes of units. Eight percent were excited by stimulation of the contralateral ear, inhibited by stimulation of the ipsilateral ear (EI), and insensitive to binaural phase differences. Units were also recorded that responded (excited) only to stimulation of the contralateral ear (EO). These strictly monaural units represented only $12 \%$ of the total units recorded from the fiber bundle. Phase-sensitive units were usually found in the anterior region of the output bundle, whereas phaseinsensitive units were usually located in the more posterior region. The latencies of phase-sensitive responses averaged about $3.3 \mathrm{msec}$, ranging from 2.5 to $4.3 \mathrm{msec}$.

Nucleus ventralis lemnisci lateralis (VLV). The 297 units recorded in this area (Fig. 6) fell into two distinctly different classes. These classes differed from each other in their monaural response properties, their sensitivity to IIDs, and their sensitivity to binaural phase differences.

Figure 7 illustrates the types of responses obtained
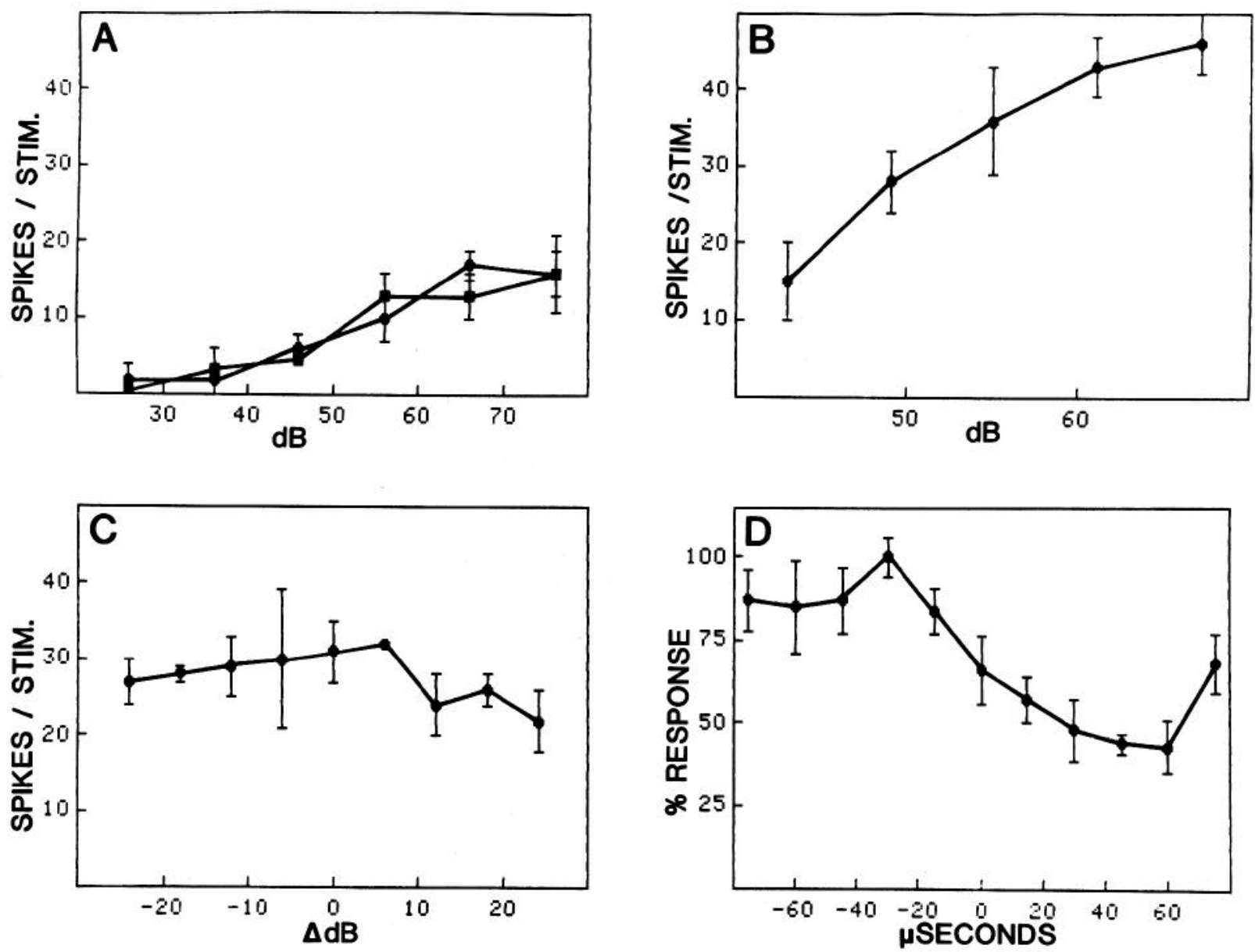

Figure 4. Example of a phase-sensitive unit from nucleus laminaris. $A$, The unit was excited by monaural stimulation of either ear ( $\bullet$, contralateral; $\mathbf{0}$, ipsilateral). $B$, Response to variations in the $\mathrm{ABI}$ (IID $=0, \mathrm{OTD}=-30 \mu \mathrm{sec}$ ). $C$, Response to variations in IID ( $\mathrm{ABI}=56 \mathrm{~dB}, \mathrm{OTD}=-30 \mu \mathrm{sec}$ ). Negative values indicate that ipsilateral stimulation was louder. $D$, The response to binaural stimulation was affected by the binaural phase difference. Negative values indicate that the stimulus delivered to the contralateral ear was leading. Stimulus: bandpassed noise. Stimulus repetitions: $5(A$ to $C)$ and $10(D)$. Spontaneous level: 1.6 spikes/stimulus $(4 \%$ in $D)$. 


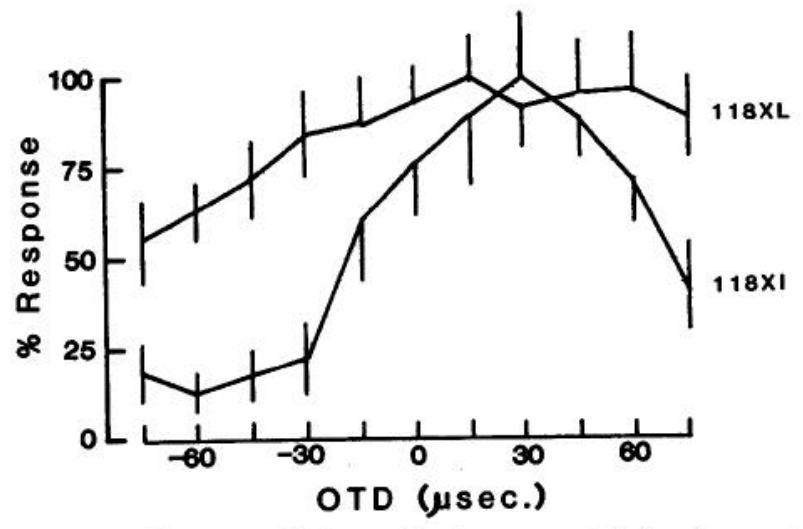

Figure 5. Range of binaural phase sensitivity in nucleus laminaris units. Sample phase sensitivity curves from two EE units representing the range of sensitivity found. Some error bars were removed to eliminate confusion (in all cases, error bars were symmetric about the mean). Stimulus: bandpassed noise (top curve), $5.9-\mathrm{kHz}$ tone bursts (bottom curve), IID $=0$ (both curves); repetitions: 12 (top) and 15 (bottom).

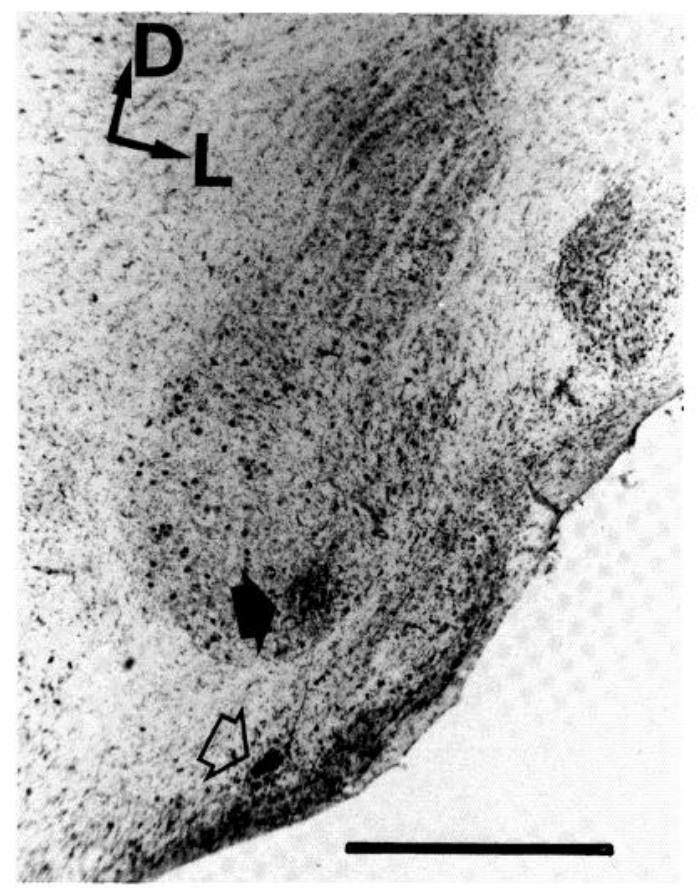

Figure 6. A coronal section showing VLV. Arrows show two electrolytic lesions in one electrode track. Open arrowhead, Lesion near the bottom of VLVa, where EE phase-sensitive units were recorded. VLVp is also visible in this section as the elongated area dorsal to VLVa. Solid arrowhead, Lesion ventral to VLVa. Calibration bar $=1.0 \mathrm{~mm}$.

from $42 \%$ of the units in this area. Stimulation of either ear excited these units (Fig. $7 A$ ). In the example shown, the two monaural inputs had equal effects on the unit. The monaural inputs were not always "balanced" in this way; some units were more sensitive to stimulation of one ear than to stimulation of the other. Their spike counts were proportional to the ABI (Fig. $7 B$ ) and were largely unaffected by variation in IIDs (Fig. $7 C$ ), providing that the intensity of sound delivered to each ear was above threshold. All of these EE units responded pref- erentially to a discrete range of binaural phase disparity (Fig. $7 D$ ). These units responded at an average latency of about $3.9 \mathrm{msec}$ (range 3.5 to $4.5 \mathrm{msec}$ ).

The other class of units found in VLV comprised $49 \%$ of the total. Stimulation of the contralateral ear excited these units and ipsilateral stimulation inhibited them (Fig. $8 A$ ). Since these units usually exhibited spontaneous activity, the inhibitory effect was easily seen. The relative strengths of the excitatory and inhibitory inputs varied among the units recorded. The spike count increased monotonically with increasing ABI (Fig. $8 B$ ). The effect of inhibition is evident when the response to binaural stimuli is compared to the monaural response function (Fig. 8A). For any given intensity, stimulation of the contralateral ear (excitatory input) alone resulted in a greater number of spikes than did binaural stimulation with the same intensity at the contralateral ear. Because the response was proportional to the algebraic summation of the excitatory and inhibitory inputs, the response of these units was a function of the IID (Fig. $8 C)$ ). The spike count was low when the inhibitory input was stronger than the excitatory one, and, similarly, the spike count was high when the excitatory input was stronger than the inhibitory one. The number of spikes did not uniquely encode the IID. Since the spike count was also proportional to the ABI (Fig. $8 B$ ), a given spike count could be obtained for different combinations of ABIs and IIDs. These units were insensitive to binaural phase differences (Fig. $8 D$ ). The approximate response latency was $4.9 \mathrm{msec}$ (range 3.4 to $6.6 \mathrm{msec}$ ).

The two classes of units recorded in this area occurred in two anatomically distinct regions: EE units which were sensitive to phase differences were located in the anterior division of VLV (VLVa) and EI units were located in the posterior division of VLV (VLVp).

Nucleus lemnisci lateralis pars ventralis ( $L L v)$. All of the 118 units recorded in LLv were monaural. They received excitatory input from the contralateral ear and no input from the ipsilateral ear (EO). They were also presented with the standard binaural stimulus protocol but exhibited no evidence of binaural sensitivity.

Nucleus mesencephalicus lateralis, pars dorsalis $(M L D)$. This nucleus is the avian homologue of the inferior colliculus. It consists of three cytoarchitectonically distinct subdivisions: the central, external, and superficial nuclei. The central nucleus receives its input directly from the pontine and medullary auditory nuclei, whereas the external nucleus has no direct connections with them (Knudsen, 1983a). Space-specific units of the types $\mathrm{OO}$, IO, OI, and II occur exclusively in the external nucleus. For the purpose of the present report, we will describe only the physiological properties of space-specific units, although other types of units occur in other subdivisions.

Space-specific units could not be excited by monaural stimulation of either ear. When spontaneously active, they could be inhibited by monaural input. Units which were not spontaneously active did not show any response to monaural input (presumably, they were inhibited, but there was no way to test this). Variations in the ABI had little effect on their response (Fig. 9A). However, when the IID was varied and the average intensity was kept constant (Fig. 9B), their response peaked sharply within 

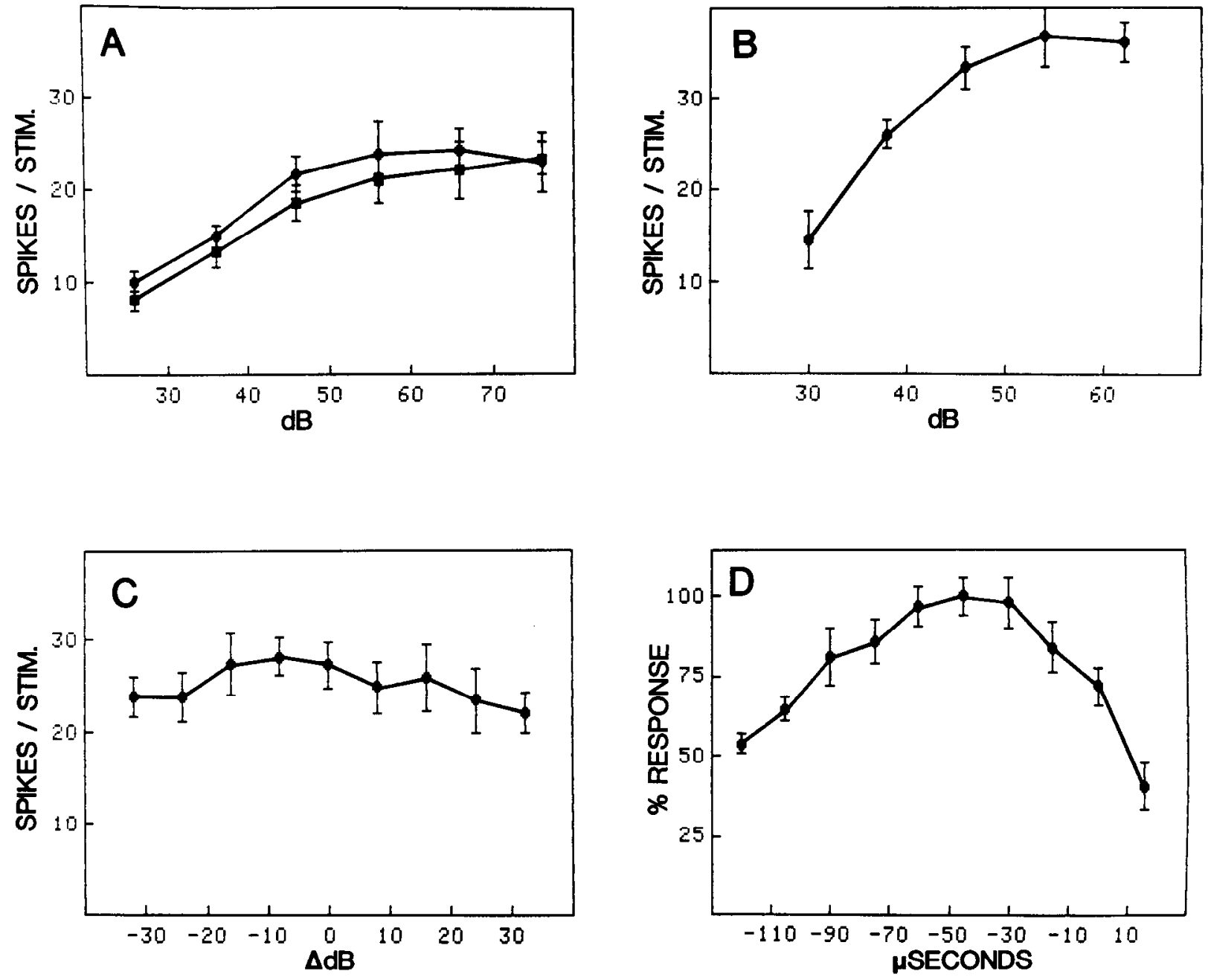

Figure 7. Responses of a typical phase-sensitive VLVa unit. $A$, Unit was excited by monaural stimulation of either the contralateral (circles) or the ipsilateral (squares) ears. $B$, Effect of varying the ABI (IID $=0,0$ TD $=$ contralateral leading by 45 $\mu \mathrm{sec}) . C$, Effect of varying the IID (ABI $=46 \mathrm{~dB}, \mathrm{OTD}=-45 \mu \mathrm{sec}$ ). Negative intensity differences indicate that ipsilateral sound was louder than contralateral sound. $D$, The response to binaural stimulation depended on the binaural phase difference. (IID $=$ $0, \mathrm{ABI}=46 \mathrm{~dB}$ ). Negative values indicate that the contralateral signal led the ipsilateral signal. This unit responded best at phase differences corresponding to the contralateral signal leading by $45 \mu \mathrm{sec}$. Stimulus: bandpassed noise. Stimulus repetitions: $8(A$ to $D)$. Spontaneous level: 6.6 spikes/stimulus ( $13 \%$ in $D)$.

a narrow range of binaural intensity differences. Thus they were "IID tuned." Their optimal intensity differences ranged from about $10 \mathrm{~dB}$ (contra $>$ ipsi) to $10 \mathrm{~dB}$ (ipsi > contra). Furthermore, effective stimuli for spacespecific units had to contain both correct intensity and phase differences, either parameter alone being ineffective (Fig. $9 \mathrm{C}$ ). Their sensitivity to both of these binaural parameters distinguished them from the brainstem neurons that were sensitive either to interaural intensity or to phase differences. Latencies of space-specific units' responses varied greatly, but were always greater than 7 msec.

\section{Discussion}

The auditory brainstem nuclei can, on the basis of the responses of their neurons to binaural stimuli (Table I), be divided into separate pathways responsible for processing either binaural phase or intensity differences. Neurons sensitive either to time (phase) or to intensity differences are segregated in the early stages of binaural interaction: nucleus laminaris is the lowest binaural nucleus containing neurons sensitive to binaural phase differences. Nucleus laminaris receives its input exclusively from nucleus magnocellularis, the cochlear nucleus specialized for retaining phase information (W. E. Sullivan and M. Konishi, manuscript in preparation). Thus, nucleus magnocellularis is the first stage of the phasesensitive pathway (Fig. 10). A subset of neurons in nucleus laminaris was sensitive to variations in binaural phase differences but not to variations in binaural intensity differences. The degree of phase sensitivity varied, but in all cases these units responded to behaviorally realistic phase differences. The maximum interaural time delay that an owl would be subject to, under natural conditions, would be about $180 \mu \mathrm{sec}$ (Moiseff and Konishi, 1981b). The magnitude of phase differences to which laminaris neurons were sensitive was within this range. The characteristics of nucleus laminaris neurons parallel those of neurons found in the homologous mammalian nucleus, the medial superior olivary nucleus (MSO). The MSO contains binaural neurons that are sensitive to 

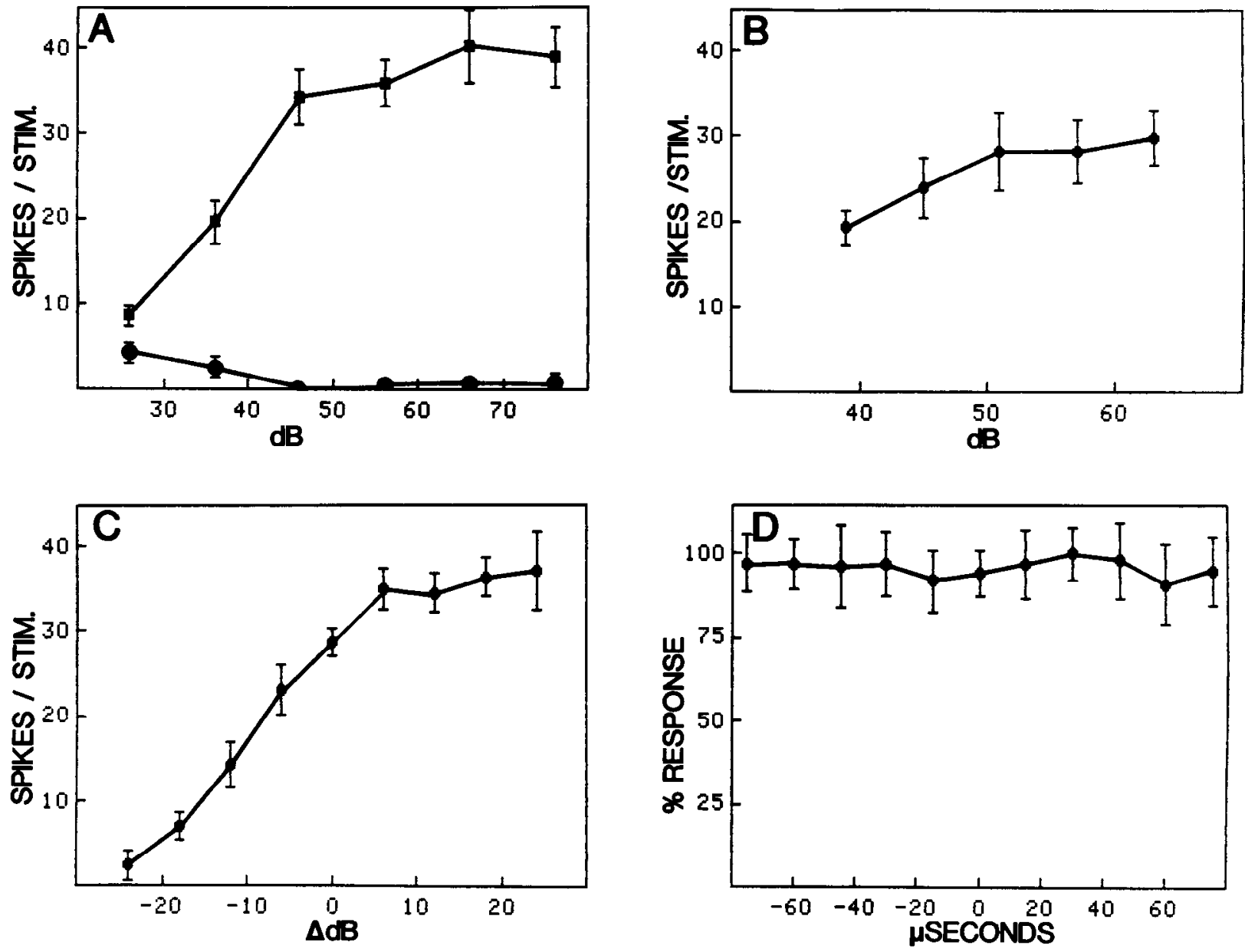

Figure 8. Responses of a typical unit from VLVp. A, Unit was excited by stimulation of the contralateral ear (squares) but was inhibited by stimulation of the ipsilateral ear (circles). $B$, Effect of varying the ABI (IID $=0,0$ TD $=30 \mu$ sec). $C$, Unit was sensitive to IIDs. Its response varied in proportion to the intensity difference. Negative $\Delta \mathrm{dB}$ indicates ipsilateral sound louder than the contralateral sound $(\mathrm{ABI}=51 \mathrm{~dB}, \mathrm{OTD}=30 \mu \mathrm{scc}) . D$, Unit was insensitive to variations in binaural phase differences (negative value $=$ contralateral side leading) $(\mathrm{IDD}=0, \mathrm{ABI}=46 \mathrm{~dB})$. Stimulus: bandpassed noise, repetitions: $8(A$ to $D)$, spontaneous level: 3 spikes/stimulus $(9 \%$ in $D)$.

interaural phase differences, and has been studied extensively (Rose et al., 1966; Moushegian et al., 1967, 1972; Goldberg and Brown, 1969; Goldberg, 1975; Inbody and Feng, 1981). Two differences stand out when comparing phase-sensitive neurons in the owl's nucleus laminaris with similar neurons in the MSO. In general, only MSO neurons with best frequencies of less than about $2 \mathrm{kHz}$ exhibit sensitivity to interaural phase differences. This contrasts sharply with the owl's phase-sensitive neurons which have best frequencies up to at least $8 \mathrm{kHz}$. The other major difference is in the range of phase (time) differences to which the neurons are sensitive. Neurons in nucleus laminaris are strongly modulated by changes in interaural phase of less than $45 \mu \mathrm{sec}$, whereas mammalian MSO neurons require much greater time differences.

Laminaris neurons project to VLVa (unpublished data). All of the neurons we recorded in VLVa were sensitive to binaural phase difference. Here, too, the best frequencies of neurons range from at least 4.5 to $8 \mathrm{kHz}$. These neurons are also unaffected by physiologically significant IIDs. These facts support the hypothesis that the pathway containing nucleus magnocellularis, nucleus laminaris, and VLVa is specialized to process phase difference information.

VLVp is the lowest binaural nucleus containing neurons sensitive to IIDs. This nucleus receives its input from nucleus angularis, which is specialized for retaining information about the stimulus intensity (W. E. Sullivan and M. Konishi, manuscript in preparation). Thus, the intensity difference-sensitive pathway consists of nucleus angularis and VLVp. Neurons in VLVp possess binaural response properties which differ markedly from those in the anterior division. The response of these neurons is a function of the excitatory effect of contralateral stimulation and the inhibitory effect of ipsilateral stimulation. Phase differences do not affect the response of these neurons. The response characteristics of neurons in this nucleus are similar to those reported for the lateral superior olivary nucleus in mammals (Tsuchitani and Boudreau, 1969; Goldberg, 1975). This nucleus, too, is composed largely of neurons excited by contralateral stimulation and inhibited by ipsilateral stimulation. The similarity between these nuclei is made more interesting 

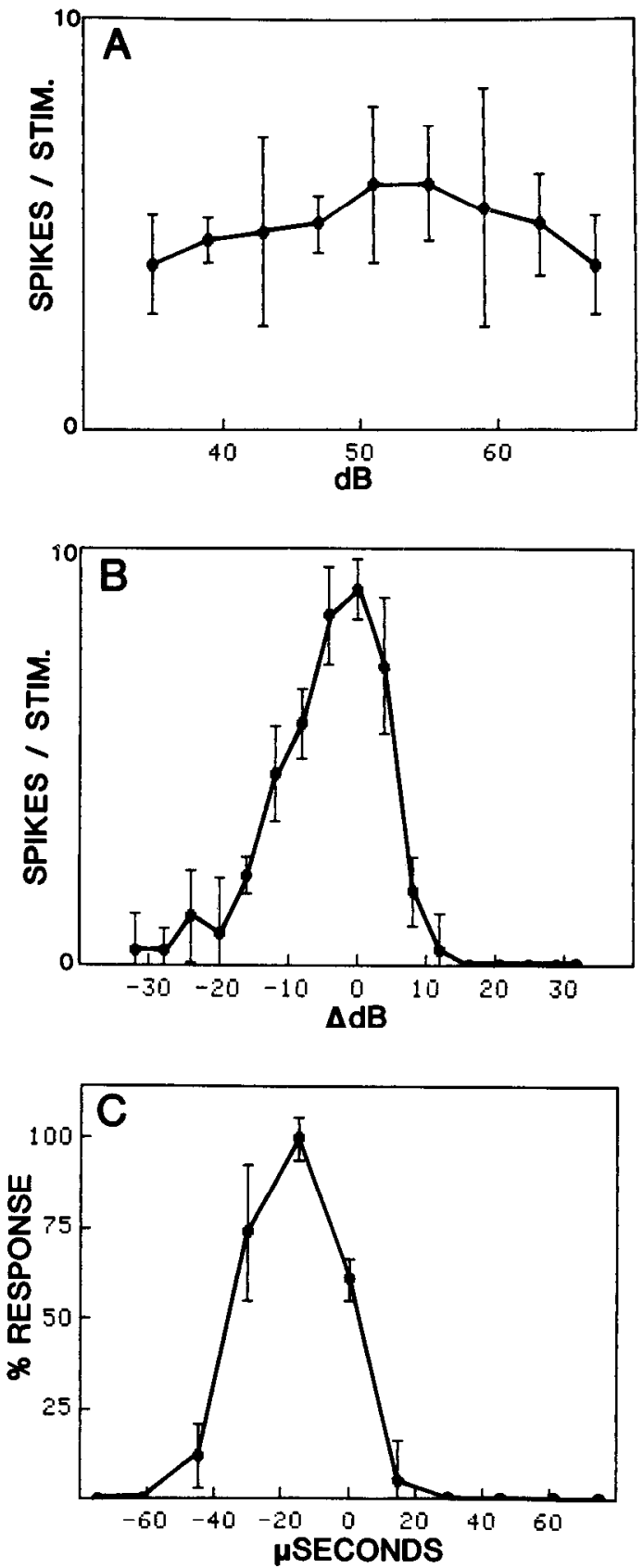

Figure 9. Example of a space-specific MLD unit. A, Unit's response was not strongly affected by variations in the ABI (IID $=0$, OTD $=-15 \mu \mathrm{sec}$ ). $B$, Unit was tuned to a narrow range of binaural intensity differences $(A B I=51 \mathrm{~dB}, \mathrm{OTD}=$ $-15 \mu \mathrm{sec})$. Negative values indicate that the contralateral sound was louder. $C$, Unit was also tuned to a narrow range of binaural phase differences (IID $=0, \mathrm{ABI}=46 \mathrm{~dB}$ ). Negative values indicate that the contralateral stimulus was leading. Stimulus: bandpassed noise. Stimulus repetitions: 5 ( $A$ to $C$ ).

by the lack of any known avian homologue for the lateral superior olive.

The midbrain auditory nucleus, the avian homologue of the inferior colliculus, is the first site in the ascending auditory pathway where space-specific neurons occur (cf. Knudsen, 1983b). Their space specificity results from their tuning to a particular combination of interaural phase and intensity differences. Deviation from the ef-

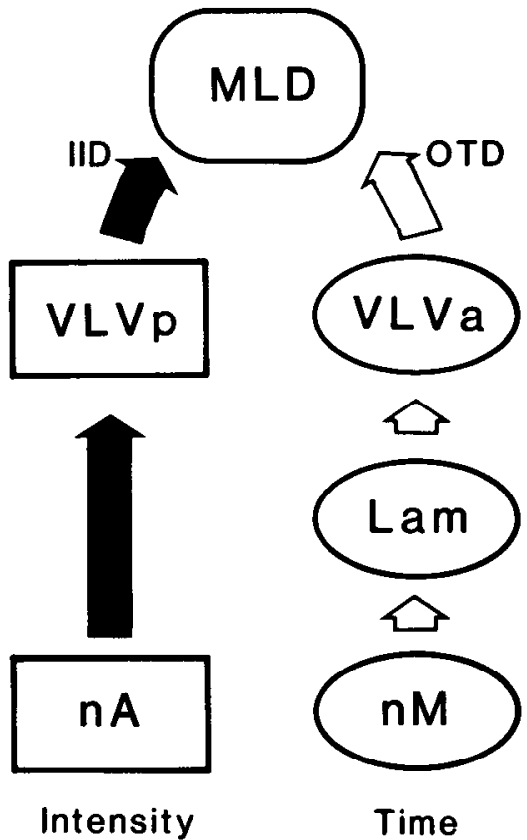

Figure 10. Binaural phase and intensity (difference) pathways through the owl's brainstem. Binaural intensity (solid arrows) and phase difference (open arrows) signals ascend the auditory pathway via two separate routes to the midbrain. Nuclei in either route contain units sensitive to variations of only one of these binaural parameters. The intensity (difference)-sensitive pathway ( $n A, V L V p)$ and the phase-sensitive pathway ( $n M$, Lam, VLVa), "IID" and "OTD," respectively, converge at the inferior colliculus $(M L D)$. Specialized units emerge in the inferior colliculus that are sensitive to both intensity and phase diferences simultaneously. Tuning to both of these parameters underlies the spatial receptive fields of these space-specific units. This diagram does not represent all of the anatomical connections between brainstem nuclei, nor does it include nuclei characterized by neurons insensitive to either phase or intensity differences. $n A$, nucleus angularis; $n M$, nucleus magnocellularis; $L a m$, nucleus laminaris, $V L V$, nucleus ventralis lemnisci lateralis ( $a$, anterior; $p$, posterior); $M L D$, nucleus mesencephalicus lateralis, pars dorsalis; $O T D$, ongoing time disparity (phase difference) sensitivity; $I I D$, interaural intensity difference sensitivity.

fective combination by as little as $10 \mu \mathrm{sec}$ or $4 \mathrm{~dB}$ can render the stimulus either ineffective or inhibitory.

The mammalian inferior colliculus, like the avian MLD, contains neurons sensitive to time or intensity differences (Rose et al., 1966; Geisler et al., 1969; Kuwada et al., 1979; Semple and Aitkin, 1979; Crow et al., 1980). Units sensitive to IIDs are generally EI, similar to the cells in VLVp (Rose et al., 1966; Geisler et al., 1969; Semple and Aitkin, 1979), but they are not "tuned" to a range of intensity differences like the owl's space-specific units. Also, neuronal tuning to a combination of binaural phase and intensity values at the level of the inferior colliculus seems, at present, to be unique to the owl.

The separation of neural pathways responsible for processing interaural time and intensity information reflects the owl's ability to divide auditory space into azimuth and elevation. The owl's external ear, made up of specialized feathers, makes the IID largely a function of stimulus elevation and the interaural time difference largely a function of stimulus azimuth. These binaural 
parameters remain segregated in the auditory brainstem. The separation must underlie this behavioral strategy.

\section{References}

Boord, R. L. (1968) Ascending projections of the primary cochlear nuclei and nucleus laminaris in the pigeon. J. Comp. Neurol. 133: 523-542.

Boord, R. L., and G. L. Rasmussen (1963) Projection of the cochlear and lagenar nerves on the cochlear nuclei of the pigeon. J. Comp. Neurol. 120: 463-475.

Crow, G., T. L. Langford, and G. Moushegian (1980) Coding of interaural time differences by some high frequency neurons of the inferior colliculus. Hear. Res. 3: 147-163.

Geisler, C. D., W. S. Rhode, and D. W. Hazelton (1969) Response of inferior colliculus neurons in the cat to binaural acoustic stimuli having wide-band spectra. J. Neurophysiol. 32: $960-974$.

Goldberg, J. M. (1975) Physiological studies of auditory nuclei of the pons. In Handbook of Sensory Physiology, W. D. Keidel and W. D. Neff, eds., Vol. V/2, pp. 109-144, Springer-Verlag, New York.

Goldberg, J. M., and P. B. Brown (1969) Response of binaural neurons of dog superior olivary complex to dichotic tonal stimuli: Some physiological mechanisms of sound localization. J. Neurophysiol. 32: 613-636.

Inbody, S. B., and A. S. Feng (1981) Binaural response characteristics of single neurons in the medial superior olivary nucleus of the albino rat. Brain Res. 210:361-366.

Karten, H. J., and W. Hodos (1967) A Stereotaxic Atlas of the Brain of the Pigeon, Johns Hopkins University Press, Baltimore.

Knudsen, E. I. (1983a) Subdivisions of the inferior colliculus in the barn owl. (Tyto alba). J. Comp. Neurol., 218: 174-186.

Knudsen, E. I. (1983b) Synthesis of a neural map of auditory space in the owl. In Dynamic Aspects of Neocortical Function, G. M. Edelman, W. M. Cowan, and W. E. Gall, eds., John Wiley \& Sons, Inc., New York, in press.

Knudsen, E. I., and M. Konishi (1978a) A neural map of auditory space in the owl. Science 200: 795-797.

Knudsen, E. I., and M. Konishi (1978b) Space and frequency are represented separately in the auditory midbrain of the owl. J. Neurophysiol. 41:870-884.
Knudsen, E. I., and M. Konishi (1982) A theory of neural auditory space. Auditory representation in the owl and its significance. In Cortical Sensory Organization. Vol. 5: Multiple Auditory Areas, C. N. Woolsey, ed., pp. 219-229, Humana Press, Clifton, NJ.

Kuwada, S., T. C. Yin, and R. E. Wickesburg (1979) Response of cat inferior colliculus neurons to binaural beat stimuli: Mechanisms for sound localization. Science 206: 586-589.

Leibler, L. (1975) Ascending binaural and monaural pathways to mesencephalic and diencephalic auditory nuclei in the pigeon Columba livia. Doctoral thesis, Massachusetts Instilute of Technology, Cambridge, MA.

Mesulam, M. M. (1978) Tetra-methyl-benzidine for HRP neurochemistry: A non-carcinogenic blue reaction product with superior sensitivity for visualizing afferents and efferents. J. Histochem. Cytochem. 26: 106-117.

Moiseff A., and M. Konishi (1981a) Neuronal and behavioral sensitivity to binaural time differences in the owl. J. Neurosci. 1: 40-48.

Moiseff, A., and M. Konishi (1981b) The owl's interaural pathway is not involved in sound localization. J. Comp. Physiol. 144: 299-304.

Moushegian, G., A. L. Rupert, and T. L. Langford (1967) Stimulus coding by medial superior olivary neurons. J. Neurophysiol. 30: 1239-1261.

Moushegian, G., A. Rupert, and M. A. Whitcomb (1972) Processing of auditory information by medial superior-olivary neurons. In Foundations of Modern Auditory Theory, J. V. Tobias, ed., pp. 265-299, Academic Press, Inc., New York.

Rose, J. E., N. G. Gross, C. D. Geisler, and J. E. Hind (1966) Some neural mechanisms in the inferior colliculus of the cat which may be relevant to the localization of a sound source. J. Neurophysiol. 29: 288-314.

Semple, M. N., and L. M. Aitkin (1979) Representation of sound frequency and laterality by units in the central nucleus of cat inferior colliculus. J. Neurophysiol. 42: 1626-1629.

Tsuchitani, C., and J. C. Boudreau (1969) Stimulus level of dichotically presented tones and cat superior olive s-segment cell discharge. J. Acoust. Soc. Am. 46: 979-988.

Wolbarsht, M. L., E. F. MacNichol, and M. G. Wagner (1960) Glass insulated platinum microelectrode. Science 132: 13091310. 